\title{
Research on the Contemporary Study of Vocal Music Art Trend from the Aesthetics Perspective and Rhythm Background
}

\author{
Lijuan Zhao \\ School Of Arts, Hubei University Of Education, \\ Wuhan,Hubei,430205 China
}

\begin{abstract}
In this paper, we conduct research on the contemporary study of the vocal music art trend from the aesthetics perspective and rhythm background. Music and art as a practice, under the current background, the modern research of music aesthetics are also constantly improve and enhance, this kind of music to explore law of the music to explain and study mode, plays an important role in the development of music of China, at the same time, increasing the current in the field of music as a whole, music performing arts with the augmentation of the research of the music aesthetics has been improved, therefore, to strengthen the basic relationship between modern music aesthetics and music performance art research, it is of great significance to the development of modern music. Our paper proposes the novel perspective on the issues that holds special significance.
\end{abstract}

Keywords-Vocal Music, Aesthetics Perspective, Rhythm Background, Contemporary Study.

\section{Introduction}

Western music is in the whole history of the development of the western society in the formation, development, maturity and evolving. As a result, the whole of western society in the political, cultural and economic development has been on the western music with the effect that cannot ignore. In the field of culture, literature, painting, construction and other sister art connected with the music. Among them, the art is the highest form of human perceptual, the music is the most complex and abstract art culture matter phase, so as the highest form of basic human rational philosophy, is bound to cultural phenomenon of human intellectually challenging the philosophical attention and thinking.

According to the literature review, the contemporary research on the music could be summarized as the following aspects. (1) Basic assumptions and premise analysis of music performing arts, review. Any scientific research is never card since the Ming assumption, in many times to self-criticism music performance art is no exception. Music aesthetics as a criterion and the metaphysical methodology framework, one of the biggest advantages is to surpass the limits of all branches of music art category. (2) Evaluation of scientific rationalism and its demonstration effect. Just like other scientific concept, many thoughts of western music performance art research is based on Newtonian physics established, on the basis of rationalism, to adhere to the symmetry of the music performance, adhere to the linear analysis. (3) Establish started from the concept of music aesthetics, has led to a music performance art and ethics and emotional debate, the former into a relatively out of the music performing arts, single performance skills, performance, music and science equilibrium model. (4) We should think western music performance art is in a crisis, and constant debate, too rational and reserve the situation of unify the whole country music performance paradigm has been broken, various views at odds [1-3]. 
Music aesthetics to promote rational and human nature, scientific spirit and humanistic spirit, value analysis and fact analysis between traditional and advantages of cooperative communication, how to music esthetics research for mediation, to achieve the reunification of the music performance art and ethical emotion and communication with recovery in the dominant position of music performing arts. Although music aesthetics is a branch of philosophy of art, but it has very broad scope and strong professional, and it is actually quite profound. As a result, many in the industry are attributed to its philosophy of music, in their ideas for the research of music aesthetics in fact equivalent to a study of music philosophy, which from the certain degree is also very reasonable. Will be connected together with music aesthetics, philosophy is a kind of relationship, personality and common among different types of things, people's aesthetic also will change as time produce certain diversity, therefore, is very important to strengthen the research of modern music esthetics, this also will improve the important means of music performance. In the figure one, we show the principles of the vocal music art.

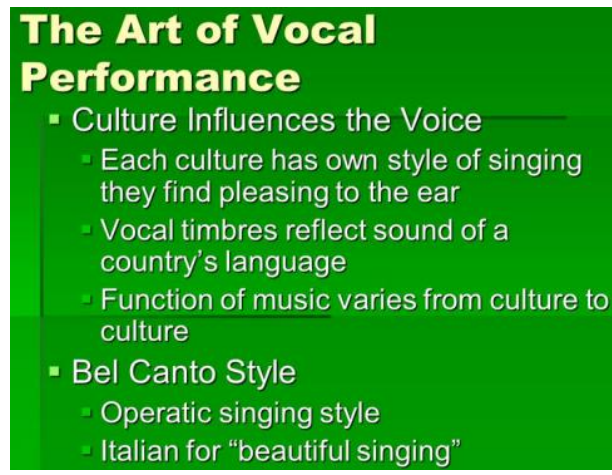

Figure 1. The Principles of the Vocal Music Art

In this paper, we conduct research on the contemporary study of the vocal music art trend from the aesthetics perspective and rhythm background. Over the past century, through the joint efforts of the several generations of the scholars that has had great progress, no matter the size, or in the process, the discipline nature of music aesthetics and its corresponding symbol has obviously, including the study of basic problem. Relative to western academic circles, the Chinese academic circle for the theories research of basic problem is prominent. As to say, why stand out in this aspect, Chinese scholars may be related to an overview of the way to use most of the economist's basic, however, it is a further study of the methodological problems of Chinese characteristics.

\section{Our Proposed Methodology}

The Property of Rhythm Background. Words and music, as a basic representative of the auditory information of two major has some similar features: they are composed of continuous events unfold over time, and by the same parameters such as pitch, schedules and loudness to indicate its phonology features as two systems has certain rules of grammar and phonetics, and apply the same set of blocks and voice stream segmentation rules forming sequence, and organized into phrases [4-5].

Ecological aesthetics and basic ecological rationality in the music of the music art is the perfect personality, from the holistic view of aesthetic education aims to inspire modern people's emotional experience, activation of the life activities, to form a scientific, free music ecological values. In fact, the whole of music aesthetic education function and the function of the ecological aesthetic education trend is consistent, also is for the human and nature, human and society, and the establishment of the harmonious relationship between humans and their triple play a role. Music aesthetic education as a kind of aesthetic education, education should be how to take care of people, and beautify the natural and social environment, calling upon the human and nature, human and social harmony and common development. Also, the music aesthetic education professional overall function to a structure of the biggest purpose is to through the art of aesthetic education to guide the human 
abandoning alienation, establish its own perfect personality that return to spiritual home.

In today's society, the music aesthetic education should reflect on their own and self-improvement, innovation breakthrough. This requires that music aesthetic education that should not only within the limitations of the narrow scope of the school education and family education, nor confined to stage performances, and more importantly, should expand their horizons, as should fully display its social education, and then a kind of life education, the integrated function of the ecological education, make it appears as a kind of spiritual life performance, or the performance is leading the people to love life. Sphere, in fact, the ecological rationality of music, experience the life spirit of actual is the integrity of the system, it is first and foremost in imprinting the running rhythm and rhythm of life, at the same time appear in people's survival activities, including people's emotional life experience, social and mental activity, and even in the deep structure of the human soul.

To understand the language and music, perception of phrases is crucial, and the process without the correct phrases identification phrase boundary. Usually these phrase boundary by the certain structure tags to characterization. Rhythm of spoken English often border through movement of fundamental frequency and the schedule information, such as boundary syllables to extend and obtain pause before insertion, these parameters are also music boundary markers [6].

The Vocal Music Art Features. Vocal music is a kind of comprehensive art form as is the perfect combination of music and literature, of which the language art is the foundation of vocal music art, if did not have the language will not be able to call vocal music, vocal music language combined with melody express and convey thoughts and feelings. Vocal language specifically through music this kind of unique artistic shape means, language rhythm of music and singing characteristic. These unique artistic languages after the creation of the composers and layout, to make the literary language, words, music, melody and rhythm in music, vocal language arts covers the field widely, but from the point of Chinese traditional vocal music, it contains drama language, the language of the opera, folk art language, folk songs and language, etc., the language types both belong to the vocal language, but because of their respective artistic performance that also have different characteristics.

\section{New Musical Forms}

- Symphonic Poem,: an orchestral work that portrayed a story or had some kind of literary or artistic background to it.

- Art Song, : a vocal musical work with tremendous emphasis placed on the text or the symbolical meanings of words within the text.

- Programme music: music that tells a story or describes a scene.

Figure 2. The New Vocal Music Art Forms

In vocal music singing art, for the purpose of vocal music art need to perfect the quality and the exquisite voice technology as the foundation, the sound is clear and bright or deep fullness, is a strong robust or crisp dainty, euphemism is a soft fruity or beautiful all show different sound quality and the basic features that could be summarized as the following aspects. (1) Vocal organs, is an organic whole. It is different from other disciplines see not want to rely on thought by feeling, after numerous repeated training. Only make the vocal organs work together properly, can make the right sound, rich and colorful and make sound wonderful. (2) Vocal music art is different from other disciplines, vocal training is based on the human body as instrument, with the training of thinking and feeling, in several times of hard training to find out the accurate sound feeling. Into a set of training thinking and feeling and this wonderful feeling of the voice that could be attained only by the voice training. (3) Correct breathing method for good when singing voice is critical from the point of the singing art, singing respiration is one 
of the important means of artistic expression from singing, breathing to provide the power, voice, feelings, to sing, singing sound accurate, sound beautiful and the expression is closely related to the breath. (4) Singing in the language, is one of the important factors in the art of singing, singing is singing songs and language to convey thoughts and feelings of an art form. So it is very important in learning vocal music singing language. About language study language pronunciation problems that should be comprehensive that is one of the important conditions of singing language pronunciation clear, but it is not the only standard, more important is language pronunciation clear at the same time, sing language to express feelings [7].

The Aesthetics Perspective. Music aesthetics is the study of human music aesthetic activities of the experience theory of the nature of discipline, is the combination of aesthetics and music is in the nature of philosophy of music basic theoretical subjects and the music aesthetics in order to study the aesthetic principles of music art as the objective. Music to create order from chaos, because general melody, rhythm conform to make continuous is better than the fracture, and harmonious compatibility is better than the not harmonious, and the resulting aesthetic feeling, produce the beauty. With all the elements of art, music elements are only to feel and to create things is a kind of beautiful. Concert to make a kind of the atmosphere, in the music concept and signals to the brain is extremely profound impression we won't be stimulated or appease such a specific function to find the source of all forms of music and music appreciation. However, this process will bring us deep aesthetic implications.

In the process of appreciation, music because of the particularity of its material and ontology is can make people to get rid of, the judgment of conceptual reason, and actively participate in the work, the intuitive to grasp the full of the personality, sensibility of true now; At the same time, it is due to the particularity of music material components and ontology exist, to make people can continue to pursue music in the music appreciation practice work, understandable that exist in the quantity now, because of the way like now quantity will make people have such the feeling, that is "seems to be closer to the music". Music is the most primitive, but it is also the highest level of art. Music education is a model of engineering, it can cultivate students have to know the world, experience life, back in the past, look to the future. It has the enlightenment of the potential experience and feelings, to inspire the people's imagination and creativity, make the person obtains sublimation of spiritual edify, personality as more important is to enhance student aesthetic consciousness that make its deeper experience of aesthetics.

The Music Art Development. Because music can make dental laboratories, the highly developed social rationality condenses appears as intuitive and flexible forms of perceptual obviously, so it can give a person feel, fantasy, and the creationary imagination ability and improve the abstract thinking, keen intelligent reasoning, enhance memory, feedback of foreign information ability. Its main role is to exercise by music people the development of imaginative thinking ability, sensitive features limbs coordinate response increased rapidly, reproductive imagination and basic creative thinking under the skilled migration, the action of one instance, make the other subjects outside of music perception and research that also has a promoting function [8].

Long-term since, our understanding of the value of music is often confined to the individual level and the social value of music is not to be concerned. Music evoke human reason does not stay on the language symbols, abstract, spiritual level, and have to deploy a set of physical activity, practice, physical level, is a kind of practical reason full of passion. The unique charm and social function of the music art is a very broad, it is able to promote the emotional communication, cultivate creativity, entertainment and other 
social aspects. Music art has a unique charm, it can arouse the curiosity of people and the beauty of the harmony of music can excavate the potential of learning more to shape healthy personality. In contemporary society, music art also has the strong and unique social value and performance to promote the harmonious coexistence between man and woman, and promote social civilization harmonious coexistence and the common development, promote the understanding and communication between countries, etc. Therefore, the development of music education is related to the goal of social harmony and development. The beauty of music and basic art can promote the harmonious development of society.

\section{Conclusion}

In this paper, we conduct research on the contemporary study of the vocal music art trend from the aesthetics perspective and rhythm background. Music is the art of development, to music in our form and genre has the concept about. We can really understand the dance music, as also only in this way, it would not have been the face of dance music fully prepared, can be fully discussed with the composer, and put forward reasonable and innovation requirements for dance music. Anyhow, dance music in the dance art will always be the most intimate partner, whether from a historical perspective, or from the perspective of development, they are interdependent, causal and fuse a special relationship, only in the clear about the special relationship between them. In the future, more related research will be conducted for optimizing the corresponding issues.

\section{References}

[1] Su, Y. A. N. G. "The Construction of Public Vocal Music Education Course in General Public University." Journal of Chongqing University (Social Science Edition) 5 (2014): 030 .

[2] Weiss, Michael W., Sandra E. Trehub, and E. Glenn Schellenberg. "Something in the way she sings enhanced memory for vocal melodies." Psychological Science 23.10 (2012): 1074-1078.

[3] Jäncke, Lutz, et al. "Verbal learning in the context of background music: no influence of vocals and instrumentals on verbal learning." Behavioral and Brain Functions 10.1 (2014): 1.

[4] Stenberg, Josh. "Christian Utz and Frederick Lau (eds), Vocal music and contemporary identities: unlimited voices in East Asia and the West." Asian Ethnicity 15.3 (2014): 410-412.

[5] Leedy, Douglas. "Historical and Regional Pronunciations in Vocal Performance." Performance Practice Review 9.2 (2012): 4.

[6] Stoeger, Angela S., and Paul Manger. "Vocal learning in elephants: neural bases and adaptive context." Current opinion in neurobiology 28 (2014): 101-107.

[7] Ishwar, Vignesh. Pitch Estimation of the Predominant Vocal Melody from Heterophonic Music Audio Recordings. Diss. Master Thesis, Universitat Pompeu Fabra, Barcelona, 2014.

[8] Perruchet, Pierre, and Bénédicte Poulin-Charronnat. "Challenging prior evidence for a shared syntactic processor for language and music." Psychonomic bulletin \& review 20.2 (2013): 310-317. 